\title{
Catherine Gidney Tending the Student Body: Youth, Health, and the Modern University
}

Toronto: University ofToronto Press, 2015. 294 pp.

\section{Christabelle Sethna}

University of Ottawa

Catherine Gidney's Tending the Student Body: Youth, Health, and the Modern University is an important contribution to the history of Canadian education, medicine, health care, and youth. Concentrating on the first sixty years of the twentieth century, Gidney tracks the shift from the university's focus on the physical health of students to its post-war emphasis on their mental health. A combination of factors - the growing acceptance of germ theory, the poor condition of First World War recruits, and the Spanish Flu pandemic — meant that universities directed their initial efforts towards safeguarding the physical health of students. They made physical examinations for incoming students compulsory, hoping to detect troublesome underlying health conditions or prevent communicable diseases from spreading. The problem of communicable diseases such as tuberculosis should not be underestimated; sick students lost time during the term, infections spread to staff and faculty, and campus facilities like infirmaries proved inadequate during outbreaks of illnesses. Understandably, medical fees for students slowly rose over time, as did the demand for more medical staff, inoculations, tests, screening programs, and better sanitation.

Physical training, believed to play a major role in improving a student's physical health, was shaped by gender role expectations. Compulsory exercises for male students reinforced military ideals of physical fitness; some universities also offered indoor and outdoor games that included gymnastics and rugby. By contrast, exercises for women were not intended to build their strength or test their stamina but to correct posture and improve mobility. Tennis and golf were preferable to hockey and basketball, because the latter were seen as men's sports. Such prescriptions were based upon anxieties about overtaxing women's reproductive biology and helped to institutionalize gender differences on campus. The institutionalization of gender differences 
led, however, to the hiring of women deans, medical advisors, physical training instructors, dieticians, doctors, and nurses, albeit at a lower rank and smaller salary than their male colleagues. Still, these professional women benefitted from a degree of financial stability and personal fulfillment, assumed leadership positions, and built networks of colleagues and friends. These networks provided women students with graduate scholarships, special lecture series on women's health, support for women's athletics, and female role models. Despite the advances that women professionals made, marriage and motherhood were still viewed as the most appropriate outcome for women students and a sign of respectable citizenship. Notably, only a tiny percentage of women professionals on campus were married, not least because there was prejudice in some universities against hiring married women.

After the end of the Second World War, tuberculosis rates decreased, thanks to aggressive testing and treatment. Campus health services increased in size, fuelled by an interest in students' mental health that was, in turn, buoyed by the inroads that mental hygiene, psychology, and psychiatry were making into the study of adolescence. Although Canadian universities lagged behind their counterparts in the United States and the United Kingdom, eventually they too assigned equal importance to students' physical and mental health, expanding campus health services to include mental health consultants who assisted students experiencing difficulties on the personal level or struggling with the demands of a university education. The expansion of medical and hospital insurance coverage also played a part. The emphasis on mental health marched in step with a new discourse on personality development. Whereas building character had been seen as essential to citizenship based on Christian principles, now self-realization was key. This transition, which coincided with the secularization of Canadian society, would set the stage for a rights revolution in which women on and off campus would figure prominently from the late 1960s onwards.

Gidney's book should be seen as a starting point for scholars who wish to expand upon her findings. Although she claims to represent regional diversity, her analysis reads rather uniformly. A measured comparison among universities in terms of their age, size, specialization, regional influence, types of health services, and funding for those services would have been helpful. So too would concrete information on the ebb and flow of student enrolment, which is particularly important in regard to the population of women students. On that note, a deeper exploration of the perceived incompatibility of women and higher education is required, an incompatibility that continues to dog women into the present day if gender gaps in salary, tenure, and promotion, not to mention sexual violence on campus, are any indication. Finally, Gidney needed to engage more profoundly with the complex relationship of the university to militarism, elitism, whiteness, and nation building. Her analysis of universities that were relatively homogeneous (in terms of student and faculty racial, religious, and class backgrounds) deviates only occasionally into the more tantalizing territory of difference, aside from gender.

Despite these concerns, the book makes for particularly interesting reading today, given legal obligations that universities furnish students with an ever-widening range of services from physical and mental health care to accommodation for a variety 
of disabilities. One is struck by the sense that universities have evolved to provide for students' physical and mental health as much as for their intellect. The rights revolution with which Gidney ends her book continues to permeate the campus. However, the longstanding in loco parentis role of the university that students active in that revolution rejected seems to have made a comeback. Current pressures on cash-strapped universities to welcome a student clientele with safe spaces, sensitivity training, and trigger warnings may give credence to accusations that parents, educators, and administrators have colluded, not to promote student self-realization, but to infantilize a generation of adolescents for the twenty-first century. 